\title{
Pathogénie du diabète insulinodépendant, maladie polygénique d'origine auto-immune
}

Le diabète insulinodépendant est une maladie autoimmune à forte composante génétique. L'un des loci de susceptibilité est localisé dans le complexe majeur d'histocompatibilité et correspond probablement à des gènes codant pour les molécules de classe II, notamment DQ. La localisation du ou des autre(s) gène(s) de susceptibilité est inconnue. Le développement du diabète semble lié à des lésions d'insulite constituées de l'infiltration des îlots de Langerhans par des cellules $T$. Bien qu'il existe dans cette forme de diabète des anticorps aussi bien que des lymphocytes $T$ cytotoxiques anti-îlots, seuls ces derniers sont capables, par transfert passif, d'induire un diabète chez des receveurs non diabétiques. On ne sait pas, à ce jour, si l'élément déclenchant de la maladie est l'apparition (ou l'activation) première de cellules $\mathbf{T}$ autoréactives, un déficit en cellules $\mathbf{T}$ suppressives, ou bien si un facteur d'environnement, par exemple une affection virale, est initialement en cause.

\section{Christian Boitard Jean-François Bach}

\section{ADRESSE}

C. Boitard : docleur en médecine, professeur d'immunologie. J.-F. Bach : docleur en médecine, professeur d'immunologie. Inserm U.25 et Service d'immunologie clinique, hôpital Nec- e diabète insulino-dépendant (DID) est une maladie génétique partiellement héréditaire : $10 \%$ des diabétiques ont un antécédent diabétologique familial direct. La survenue de la maladie est contrôlée par plusieurs gènes dont le principal est situé dans le complexe majeur d'histocompatibilité $(\mathrm{CMH})$. L'environnement joue également un rôle important puisque le taux de concordance pour la maladie entre jumeaux monozygotes ne dépasse pas $40 \%$. Divers facteurs étiologiques ont été incriminés. Le rôle de virus a été évoqué avec insistance mais jamais démontré. L'intervention de toxiques a été suspectée chez quelques malades isolés mais n'a pas été confirmée sur de larges séries. Tout laisse penser, en fait, que le DID est une maladie auto-immune due à la destruction des cellules $\beta$ productrices d'insuline par des lymphocytes $T$ qui infiltrent les îlots de Langerhans. Cette origine immunologique n'exclut pas cependant, en amont, l'intervention de facteurs étiologiques non immunologiques déclenchant la réaction auto-immune.

Les arguments sur lesquels repose ce modèle pathogénique proviennent pour partie d'observations faites sur le DID humain (anticorps anti-îlots, insulite, amélioration du diabète sous 
traitement immunosuppresseur) mais, pour une part encore plus grande, sur les données obtenues au laboratoire par l'étude des deux modèles de DID spontané, la souris NOD et le rat $\mathrm{BB}$.

\section{Modèles animaux}

Deux types de diabète peuvent être observés chez l'animal. Des diabètes spontanés sont observés sur de nombreuses espèces animales, dont celui de la souris NOD (non obese diabetic) et du rat BB (biobreeding), les plus étudiés. Des diabètes "immunologiques " peuvent également être provoqués, en particulier chez la souris, par administration d'un toxique sélectif des cellules $\beta$, la streptozotocine, injectée à faibles doses répétées, ou par inoculation de virus (réovirus). Tant dans les modèles spontanés que provoqués, le rôle du système immunitaire, initialement évoqué devant l'existence d'une insulite et la présence d'auto-anticorps, a été établi par des expériences de transfert.

L'incidence du diabète chez le rat $\mathrm{BB}$ est indépendante du sexe et variable selon les colonies. La maladie apparaît le plus souvent entre 60 et 120 jours. Le rat BB présente, avant la survenue de la maladie, de nombreuses anomalies immunologiques : lymphopénie, déficit de l'immunité à médiation cellulaire (rejet des greffes de peau allogéniques, réactivité en culture lymphocytaire mixte et production de lymphocytes $\mathrm{T}$ cytotoxiques allogéniques ou en réponse aux mitogènes $T$, sensibilité à l'interleukine 2), associés à d'autres atteintes (thyroïde, estomac, gonades). La souris NOD, dont le diabète apparaît entre 90 et 180 jours, avec une large prédominance chez les souris femelles, ne présente pas d'anomalies immunologiques aussi évidentes. Le diabète est cependant associé à un syndrome de Sjögren.

Le transfert du diabète par les lymphocytes $\mathrm{T}$ a été obtenu dans les deux modèles. Chez le rat $\mathrm{BB}$, le transfert est obtenu par les lymphocy- tes spléniques d'animaux diabétiques au prix d'une stimulation in vitro par la concanavaline A [1]. Chez la souris NOD, le transfert est obtenu directement par l'injection de lym-

$m / s n^{\circ} 3$, vol. 7 , mars 91

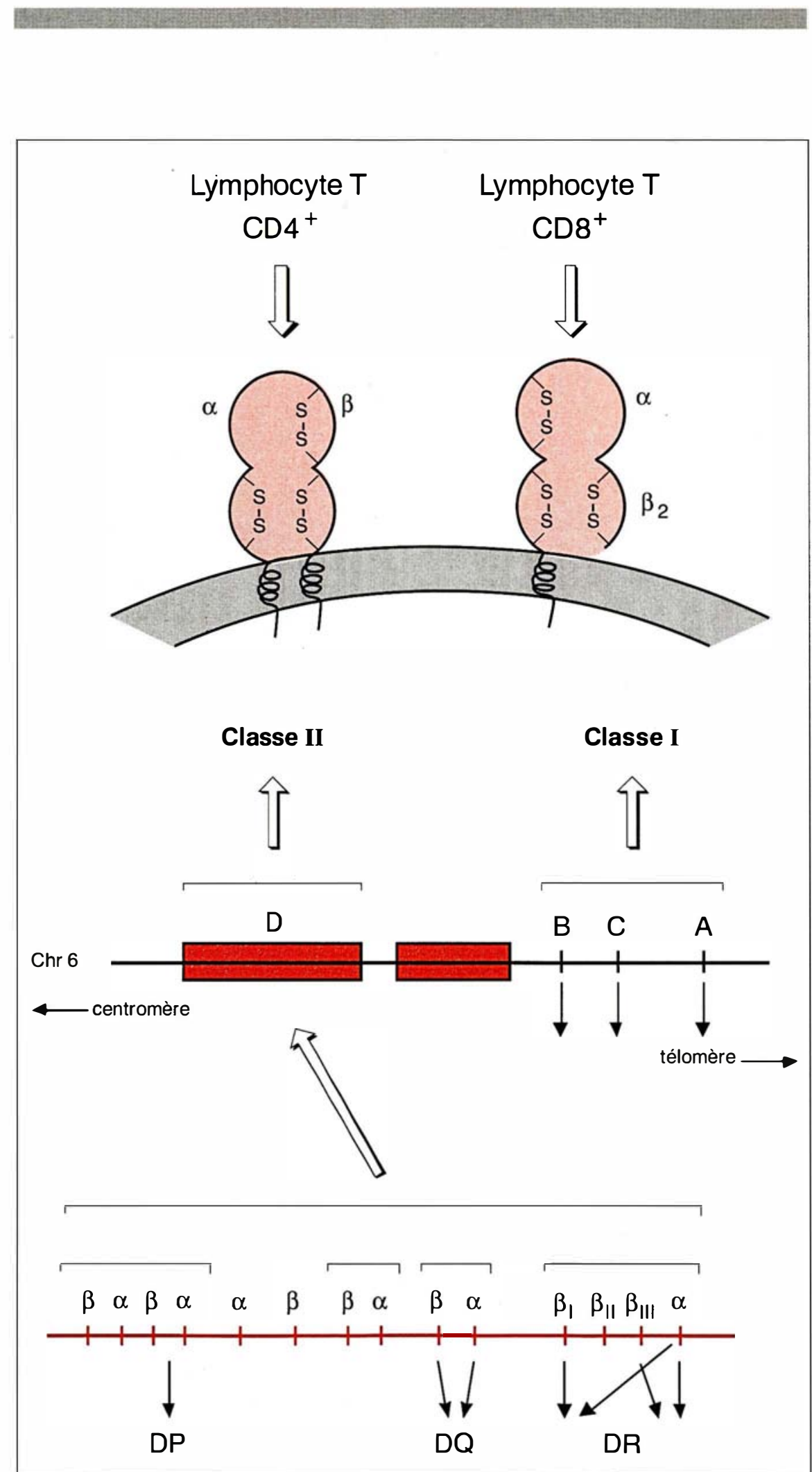

Figure 1. Le complexe majeur d'histocompatibilité. Les flèches situées sous la carte des régions $D P, D Q$ et $D R$ représentent les gènes actifs : un seul couple $\alpha \beta$ serait exprimé pour $D P$ et $D Q$, alors que, pour $D R$, le seul gène $\alpha$ pourrait s'associer soit à $\beta_{l}$, soit à $\beta_{I I !}$. 


\section{RÉFÉRENCES}

1. Like AA, Weringer EJ, Holdash A, McGill P, Atkinson D, Rossini A A. Adoptive transfer of autoimmune diabetes mellitus in biobreeding/Worcester (BB/W) inbred and hydrid rats. I Immunol 1985; 134 : 1583-7.

2. Miller BJ, Appel MC, O'Neil JJ, Wicker LS. Both the $\mathrm{Lyt} 2{ }^{+}$and $\mathrm{L} 3 \mathrm{~T}^{+}{ }^{+} \mathrm{T}$ cell subsets are required for the transfer in nonobese diabetic mice. J Immunol 1988 ; 140: 52-8.

3. Bendelac A, Carnaud C, Boitard C, Bach JF. Syngeneic transfer of autoimmune diabetes from diabetic NOD mice to healthy neonates. $J$ Exp Med 1987 ; 166 : 823-32.

4. Dardenne M, Lepault F, Bendelac A, Bach JF. Acceleration of the onset od diabetes in NOD mice by thymectomy at weaning. Eur J Immunol 1989 ; 19 : 889-95.

5. Charlton B, Bacelj A, Slattery RM, Mandel TE. Cyclophosphamide induced diabetes in NOD/Wehi mice : evidence for suppression in spontaneous autoimmune diabetes mellitus. Diabetes 1989 ; 38 : 441-7.

6. Like AA, Kislauskis E, Williams RM Rossini AA. Neonatal thymectomy prevents spontaneous diabetes mellitus in the $\mathrm{BB} / \mathrm{W}$ rat. Science $1982 ; 216$ : 644-6.

7. Hahn HJ, Lucke S, Kloting I, Volk HS, Baehr RV, Diamanststein T. Curing BB rats of freshly manifested diabetes by shortterm treatment with a combination of a monoclonal anti-interleukin 2 receptor antibody and a subtherapeutic dose of cyclosporin A. Eur J Immunol 1987 ; 17 : 1075-8.

8. Shizuru J, Taylor-Edwards C, Banks BA, Gregory AK, Fathman CG. Immunotherapy of the nonobese diabetic mouse : treatment with an antibody to T-helper lymphocytes. Science 1988 ; 240 : 659-61.

9. Boitard C, Bendelac A, Richard MF, Carnaud C, Bach JF. Prevention of diabetes in nonobese diabetic mice by anti-I-A monoclonal antibodies : transfer of protection by splenic T cells. Proc Natl Acad Sci phocytes spléniques d'animaux diabétiques à des receveurs NOD mâles de 8 semaines pré-irradiés [2] ou à des receveurs non irradiés en période néonatale [3]. Le transfert nécessite la coopération de lymphocytes $\mathrm{T}$, $\mathrm{CD} 4{ }^{+}$et $\mathrm{CD} 8{ }^{+}$.

Le rôle direct du système immunitaire dans ces modèles est confirmé par l'induction ou la prévention du diabète par action sur le système immunitaire. Un diabète précoce est induit chez la souris NOD par thymectomie à 3 semaines ou par l'injection de cyclophosphamide qui détruit les lymphocytes $T$ suppresseurs. Le diabète induit par la cyclophosphamide est prévenu par le transfert de cellules mononucléées de souris NOD non diabétiques [5]. Chez le rat $\mathrm{BB}$, l'incidence du diabète augmente sous l'effet d'injections d'interleukine 2 dans certaines colonies [6]. Des traitements visant le système immunitaire préviennent la survenue du diabète spontané dans ces modèles. Le diabète du rat $\mathrm{BB}$ est prévenu par injection de sérum anti-lymphocytaire, irradiation lymphoïde, administration de glucocorticoïdes en association au sérum anti-lymphocytaire, ou administration de ciclosporine. Le diabète de la souris NOD est prévenu par la ciclosporine. Dans les deux modèles, le diabète est prévenu par la thymectomie néonatale [6].

Les anticorps monoclonaux sont un outil privilégié pour étudier in vivo le rôle des sous-populations lymphocytaires dans le développement de la maladie. Le diabète du rat $\mathrm{BB}$ est prévenu par l'injection in vivo d'anticorps monoclonaux dirigés contre le récepteur de l'interleukine 2 (IL-2) [7] ou les antigènes de classe II du CMH. Dans le cas du traitement par l'anticorps antirécepteur de l'IL-2, la prévention du diabète est obtenue en associant un traitement par ciclosporine à la dose de $1,5 \mathrm{mg} / \mathrm{kg}$ durant 10 jours et se maintient à long terme. De même, le diabète spontané de la souris NOD est prévenu par l'injection d'anticorps anti-CD4 + [8] ou anti-classe II [9]. Le rat $\mathrm{BB}$ et la souris NOD représentent des modèles privilégiés pour l'obtention de clones de lymphocytes $\mathrm{T}$ au cours du diabète. Des clones de lymphocytes $T$ spécifiques d'antigènes insulaires exprimés par les cellules de l'insulinome Rin5F ont été obtenus chez le rat BB [10]. Différents clones $\mathrm{T}$ ont été obtenus chez la souris NOD [11-13]. Leur injection in vivo permet soit d'induire un diabète chez des animaux indemnes, soit de prévenir la survenue du diabète chez les animaux susceptibles. L'obtention de clones spécifiques est non seulement importante dans la compréhension des mécanismes physiopathologiques impliqués dans le développement du diabète, mais aussi dans la recherche de nouvelles approches du traitement de la maladie.

Un dernier type de modèle susceptibles de contribuer à la compréhension des mécanismes physiopathologiques en jeu au cours du DID est fondé sur l'obtention de souris transgéniques utilisant des constructions couplant des séquences codant pour différents antigènes avec le promoteur de l'insuline. Il permet l'expression sélective et précoce du transgène sur les cellules $\beta[14]\left(\mathrm{m} / \mathrm{s} n^{\circ} 7\right.$, vol 4 , p. 444). L'expression de l'antigène $T$ du virus SV40 conduit, dans certaines lignées de souris où l'expression du transgène est tardive, à l'apparition d'anticorps anti-antigène $T$ et d'une insulite [15]. L'expression précoce de l'antigène $\mathrm{T}$ induit dans d'autres lignées une tolérance immunitaire vis-à-vis de l'antigène codé par le transgène. L'analogie d'un tel modèle avec les modèles d'autoimmunité est lointaine en raison de la nature même de l'antigène dont l'expression a été obtenue. L'expression dans d'autres modèles de transgénèse d'antigènes de classe I [16] ou de classe II [17] du CMH conduit selon les cas à une absence d'anomalies des cellules $\beta$ ou à une atrophic progressive des îlots de Langerhans sans insulite et sans les critères d'une réaction auto-immune. En revanche, l'expression dans les cellules $\beta$ du gène de l'interféron $\gamma$ qui entraîne une expression anormale des antigènes de classe II provoque un diabète que l'on peut rapprocher des diabètes auto-immuns [18].

\section{Les gènes du diabète insulinodépendant}

Le DID est une maladie héréditaire : $6 \%$ des frères ou sœurs de diabéti- 
que et $5 \%$ des enfants de sujets diabétiques dévcloppent la maladie. Le contrôle génétique de la maladie fait intervenir plusieurs gènes dont seul l'un est parfaitement identifié et localisé au sein du $\mathrm{CMH}$.

Les gènes de prédisposition du CMH. Les antigènes codés par le $\mathrm{CMH}$ (système HLA chez l'homme) jouent un rôle central dans les interactions moléculaires qui assurent la coopération entre cellules lymphoïdes au cours des réponses immunitaires. On distingue les antigènes de classe I $(A, B$ ou $C$ ), exprimés sur les membranes de toutes les cellules nucléées, et les antigènes de classe II (DR, DP ou DQ), exprimés dans les conditions physiologiques par les seuls lymphocytes B, les lymphocytes $T$ activés et les cellules présentant l'antigène (monocytes et macrophages). Le polymorphisme des antigènes du $\mathrm{CMH}$ a permis l'étude de leur ségrégation au sein de familles comportant plusicurs sujets diabétiques et les études de populations de sujets diabétiques ont permis de définir une forte association diabèteHLA. Les études initiales réalisées avec des marqueurs sérologiques ont montré que le DID se développe dans plus de $90 \%$ des cas chez des sujets porteurs des antigènes de classe II HLA DR3 et/ou DR4. Dans les populations caucasoïdes, les sujets DR3 ont une probabilité de 3 à 4 fois supérieure, les sujets DR4 de 6 à 7 fois supérieure à celle des sujets non DR3 ou non DR4 de développer un diabètc insulinodépendant. Des antigènes à risque différent ont été retrouvés dans d'autres populations, par exemple DR8 au Japon. L'association diabète-HLA est particulière en raison de l'effet protecteur de certains gènes (notamment DR2) qui conferent unc résistance vis-à-vis de la maladie. Une scconde particularité tient au risque élevé ( $x$ 40) lié à la présence à l'état hétérozygote de HLA-DR3 et DR4 [19]. La disponibilité récente de marqueurs sérologiques des allèles DP et DQ a permis de compléter ces données. L'étude des allèles DP a donné lieu à des résultats contradictoires. En revanche, unc association étroitc avec DQ a été suggéréc sur la base de l'utilisation d'anticorps monoclonaux

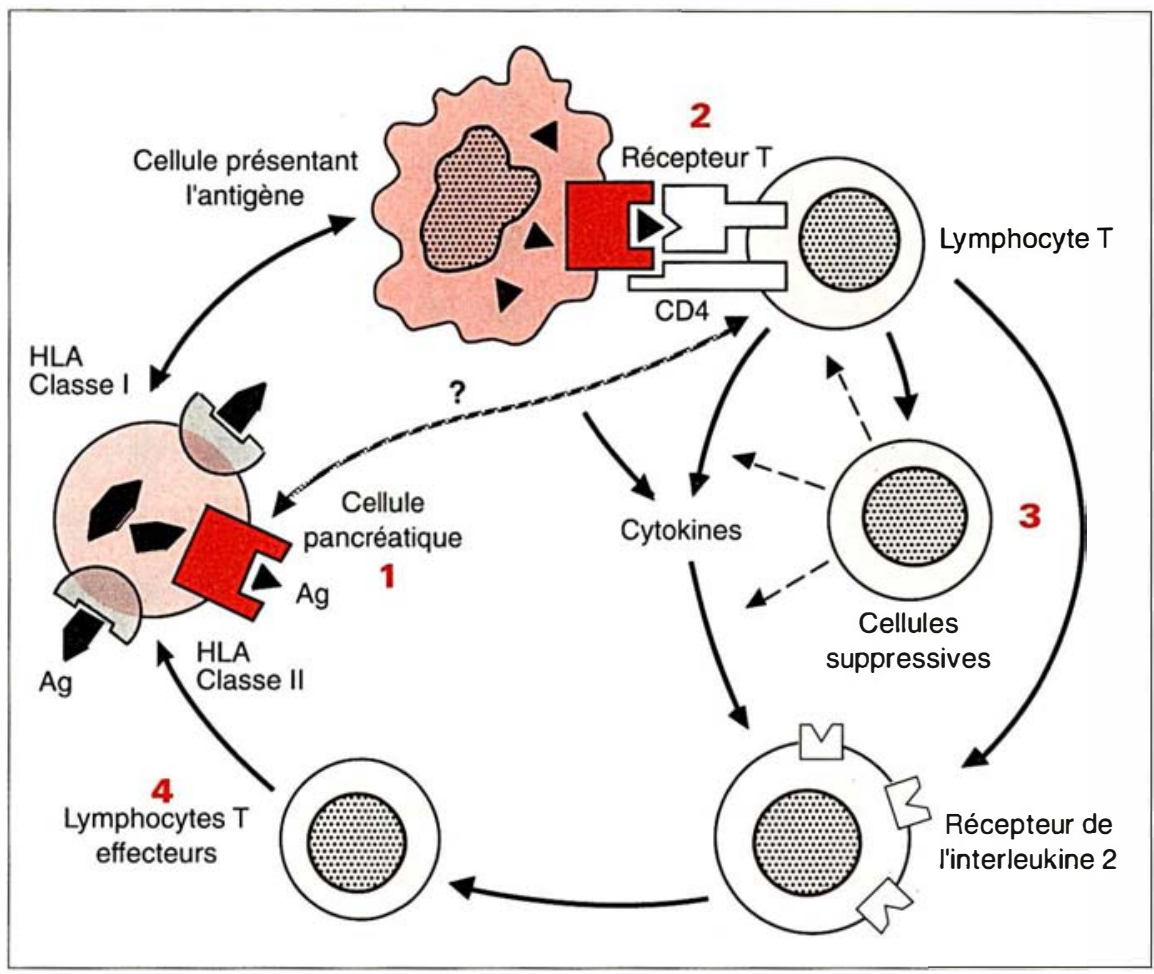

Figure 2. La réaction autoimmune dirigée contre la cellule $\beta$. Les chiffres identifient les étapes de la réaction immunitaire essentielles à la compréhension du mécanisme immunologique conduisant à la destruction des cellules. 1 - La réaction autoimmune est-elle initialement une maladie de la cible? L'identification du ou des autoantigènes cibles (Ag), une anomalie de l'expression d'un autoantigène ou de sa présentation au système immunitaire, l'expression d'antigènes de la classe II (molécules de présentation de l'autoantigène) directement par les cellules $\beta$ sont autant d'hypothèses susceptibles d'expliquer le déclenchement de la réaction autoimmune. 2 - L'interaction cellule $T$ cellule présentant l'antigène et les structures membranaires impliquées dans cette interaction sont au centre de l'activation de la réaction autoimmune. Le clonage des lymphocytes T CD4 et la caractérisation moléculaire des structures membranaires d'interaction devront être précisées. 3 - Le rôle des cellules suppressives, une anomalie de régulation des clones autoréactifs, sont susceptibles de participer au déclenchement de la réaction autoimmune. Le clonage des cellules régulatrices, la caractérisation moléculaire de leurs structures d'interactions membranaires permettront de préciser leur rôle dans le développement de la réaction autoimmune. 4 - Les mécanismes effecteurs sont les mieux connus. Le rôle direct de lymphocytes $T$ est démontré chez l'animal. La possibilité d'autres mécanismes effecteurs (anticorps, cytokines...) demeure à évaluer.

reconnaissant des formes allotypiques de la chaîne $\beta$ de $\mathrm{DQ}$.

Les spécificités sérologiques ont pu être subdivisées par typage cellulaire (capacité de stimuler in vitro des lymphocytes présentant une spécificité différentc bien que non reconnue sérologiquement) et l'étude du polymorphisme des fragments de restriction (RFLP) de l'ADN par des sondes d'ADNc spécifiques. Le typage cellulaire qui définit les spécificités Dw, corrélées à des différences portant sur la chaînc DR $\beta 1$, n'a pas permis d'établir un risque particulier au cours du diabète. Les études de RFLP ont en revanche montré l'association du diabète à certains allèles DQ [20]. Trois antigènes $D Q$ majcurs ont été initialement définis par la sérologic (DQw1, 2 et 3 ). Les études par RFLP ont subdivisé 


\section{RÉFÉRENCES}

10. Prud'homme GJ, Fuks A, Guttmann RD, Colle E. T cell hybrids with specificity for islet cell antigens. J Immunol 1986 ; 136 : 1535-6.

11. Haskins K, Portas M, Bergman B, Lafferty $\mathrm{K}$, Bradley $\mathrm{B}$. Pancreatic islet-specific $\mathrm{T}$-cell clones from nonobese diabetic mice. Proc Nall Acad Sci USA 1989 ; 86 : 8000-4.

12. Reich EP, Sherwin RS, Kannagawa O, Janeway Jr CA. An explanation for the protective effect of the MHC classe II I-E molecule in murine diabetes. Nature 1989 ; 341: 326-8.

13. Elias D, Markovits D, Reshef T, Van der Zee R, Cohen IR. Induction and therapy of autoimnune diabetes in the nonobese diabetic (NOD/Lt) mouse by a 65-kDa heat shock protein. Proc Natl Acad Sci USA 1990 ; 87: 1576-80.

14. Benoist $C$, Gerlinger $P$, LeMeur $M$, Mathis D. Transgenic mice : " new wave " immunogenetics. Immunol Today 1986: 7 : 138-41.

15. Adams TE, Alpert S, Hanahan D Non-tolerance and autoantibodies to a transgenic self antigen expressed in pancreatic $\beta$ cells. Nature 1987: 325: 223-8.

16. Allison J, Campbell IL, Morahan G Mandel TE, Harrison LC, Miller JFAP. Diabetes in transgenic mice resulting from over-expression of class I histocompatibility molecules in pancreatic $\beta$ cells. Nature 1988 ; 333 : 529-33.

17. Böhme J, Haskins $K$, Stecha $P$, et al. Transgenic mice with I-A on islet cells are normoglycemic but immunologically intolerant. Science 1989 : 244 : 1179-83.

18. Sarvetnick N, Shizuru J, Liggitt D, et al. Loss of pancreatic islet tolerance induced by $\beta$-cell expression of interferon- $\gamma$. Nature 1990 : 346: 844-7.

19. Svejgaard A, Platz P, Ryder LP. Insulin-dependent diabetes mellitus. Report of the 8th International Histocompatibility Workshop held in Los Angeles, California
DQw3, qui définit une spécificité particulière de la chaîne $\beta$, en DQw3.1, 3.2 et 3.3. Les sujets DR4 sont DQw3.1, DQw3.2 ou DQ blanc. Les sujets DR4 DQw 3.2 sont ceux qui développent un diabète. C'est également le cas de certains sujets DR2 (DQw1-AZH) ou DR1 (DQw1.1). L'obtention de la séquence nucléotidique du domaine externe de plusieurs allèles $\mathrm{DQ}$ a récemment permis de préciser ces données. Une particularité biochimique commune aux allèles associés à la susceptibilité vis-à-vis du diabète (DR1-DQw1.1, DR4-DQw3.2, DR3-DQw2, DR2-DQw1-AZH) est la présence d'une sérine, d'une alanine ou d'une valine en position 57 sur la chaîne $D Q \beta$ et non d'un acide aspartique, caractéristique des allèles non associés au diabète [21]. L'amino-acide en position 57 ne saurait résumer à lui seul la configuration moléculaire attachée au diabète mais est une première étape importante de sa définition. Un travail récent, à confirmer, a insisté sur l'importance du résidu Arg en 52 sur la chaîne $\mathrm{DQ} \alpha$ qui est obligatoircment associée à la chaîne $\beta$ [22]

Plusieurs hypothèses sous-tendent le rôle des antigènes de classe II dans le développement des maladies autoimmunes. Les observations précédentes ne peuvent exclure la présence, au sein du $\mathrm{CMH}$, de gènes de susceptibilité ne codant pas pour des antigènes de classe II mais proches (en déséquilibre de liaison) de ceuxci. La présence du gène de certaines maladies (déficit en 21-hydroxylase) est ainsi observée au sein du CMH en l'absence de toute arrière-pensée immunologique. Le rôle des antigènes de classe II dans la régulation de la réponse immunitaire met néanmoins en avant d'autres hypothèses [21]. Les antigènes de classe II sont au centre de l'interaction entre lymphocyte T CD4 + et cellules présentant l'antigène. Les gènes qui les codent fonctionnent comme des gènes de réponse immunitaire, déterminant la capacité de réponse vis-à-vis d'antigènes polypeptidiques simples. Ces gènes pourraient ainsi contrôler la réponse vis-à-vis d'auto-antigènes. L'obtention récente d'antigènes de classe I humains sous forme cristallisée conduit à un modèle structural susceptible d'expliquer le rôle des antigènes du $\mathrm{CMH}$. Le récepteur antigénique du lymphocyte $T$ reconnaît le déterminant peptidique de l'antigène dont il est spécifique dans une poche formée par l'antigène du CMH sur la membrane des cellules avec lesquelles il interagit (antigène de classe II sur les cellules présentant l'antigène pour les lymphocytes $T$ $\mathrm{CD}^{+}+$, antigène de classe I sur toutes les cellules de l'organisme pour les cellules $\mathrm{CD} 8+$ ). Le récepteur antigénique du lymphocyte $T$ reconnaît ainsi simultanément des épitopes des antigènes du $\mathrm{CMH}$ et une courte séquence d'amino-acides de l'antigène dont il est spécifique. Seuls seraient capables d'induire une réponse immunitaire les antigènes capables d'une interaction biochimique avec les antigènes du $\mathrm{CMH}$ exprimés chez un individu donné. Les particularités biochimiques des antigènes de classe II associés au diabète s'intègrent aisément dans un tel schéma, l'acide aminé en 57 sur la chaîne $\beta$ déterminant la capacité de lier l'auto-antigène et de le présenter aux lymphocytes $T$.

Le seul rôle du gène codant pour $\mathrm{DQ} \beta$ ne saurait néanmoins expliquer toutes les données accumulées au cours du diabète. Le rôle possible d'autres chaînes $(\mathrm{DQ} \alpha, \mathrm{DR} \beta)$ a été souligné. Le rôle de gènes localisés dans le $\mathrm{CMH}$ à distance de la région $\mathrm{D}$ a aussi été invoqué.

Les gènes non-MHC. Les modèles animaux de diabète apportent l'indication que d'autres régions génétiques interviennent. Le $\mathrm{CMH}$ (système $\mathrm{H}-2$ chez la souris, RT1 chez le rat) est une des régions génétiques qui, comme chez l'homme, détermine la survenue du diabète chez la souris NOD ou le rat BB. La souris NOD exprime un seul antigène de classe II (I-A, équivalent de DQ de l'homme). La chaîne $A \beta$ porte une sérine en position 57 et non un acide aspartique comme les autres souches de souris de laboratoire, rejoignant en cela le modèle humain [21]. Des expériences de génétique formelle procédant au croisement de souris NOD sur des terrains génétiques classiques $(\mathrm{C} 3 \mathrm{H}$, NON, C57BL/10) suivi d'un croisement en retour des animaux F1 avec des souris NOD donnent des résul- 
tats compatibles avec la participation d'au moins deux autres régions génétiques au risque de diabète [23]. Chez le rat BB, au moins deux régions génétiques conditionnent le développement du diabète, seul un gène paraissant lié au CMH. Les études de génétique formelle indiquent que chez l'homme, comme dans les deux modèles murins, des régions génétiques autres que le $\mathrm{CMH}$ interviennent dans le développement de la maladie. On peut espérer que la disponibilité récente de sondes reconnaissant des régions génétiques polymorphes au sein de l'espèce, autorisant une véritable cartographie du génome, permettront l'identification des autres régions génétiques de susceptibilité.

\section{Le rôle de l'environnement}

L'induction d'un diabète par des facteurs de l'environnement, viraux ou chimiques, est démontrée chez des souris normales. Le rôle de l'environnement dans le déclenchement du diabète humain reste en revanche mal étayé. Les observations de diabète à la suite d'épidémies virales (oreillons, rubéole, infections à virus coxsackie, mononucléose infectieuse, infections à cytomégalovirus), la recrudescence saisonnière des nouveaux cas de diabète, rappelant celle de maladies virales communes, sont des arguments indirects en faveur du rôle de virus. Le rôle de facteurs toxiques a été suggéré chez l'homme, mais reste mal établi. De tels facteurs, en provoquant la libération d'auto-antigènes insulaires, sont susceptibles d'activer secondairement le système immunitaire. Les relations possibles entre des facteurs diabétiques et le système immunitaire ou l'expression d'auto-antigènes exprimés sur la membrane des cellules $\beta$ soulignent enfin le rôle possible de facteurs alimentaires, également démontré chez l'animal.

\section{Le rôle central des cellules $T$}

Des arguments convergents indiquent la responsabilité exclusive ou au moins prédominante des cellules $\mathrm{T}$ dans la pathogénie du DID chez la souris NOD, chez le rat BB et chez l'homme. Le diabète de la souris

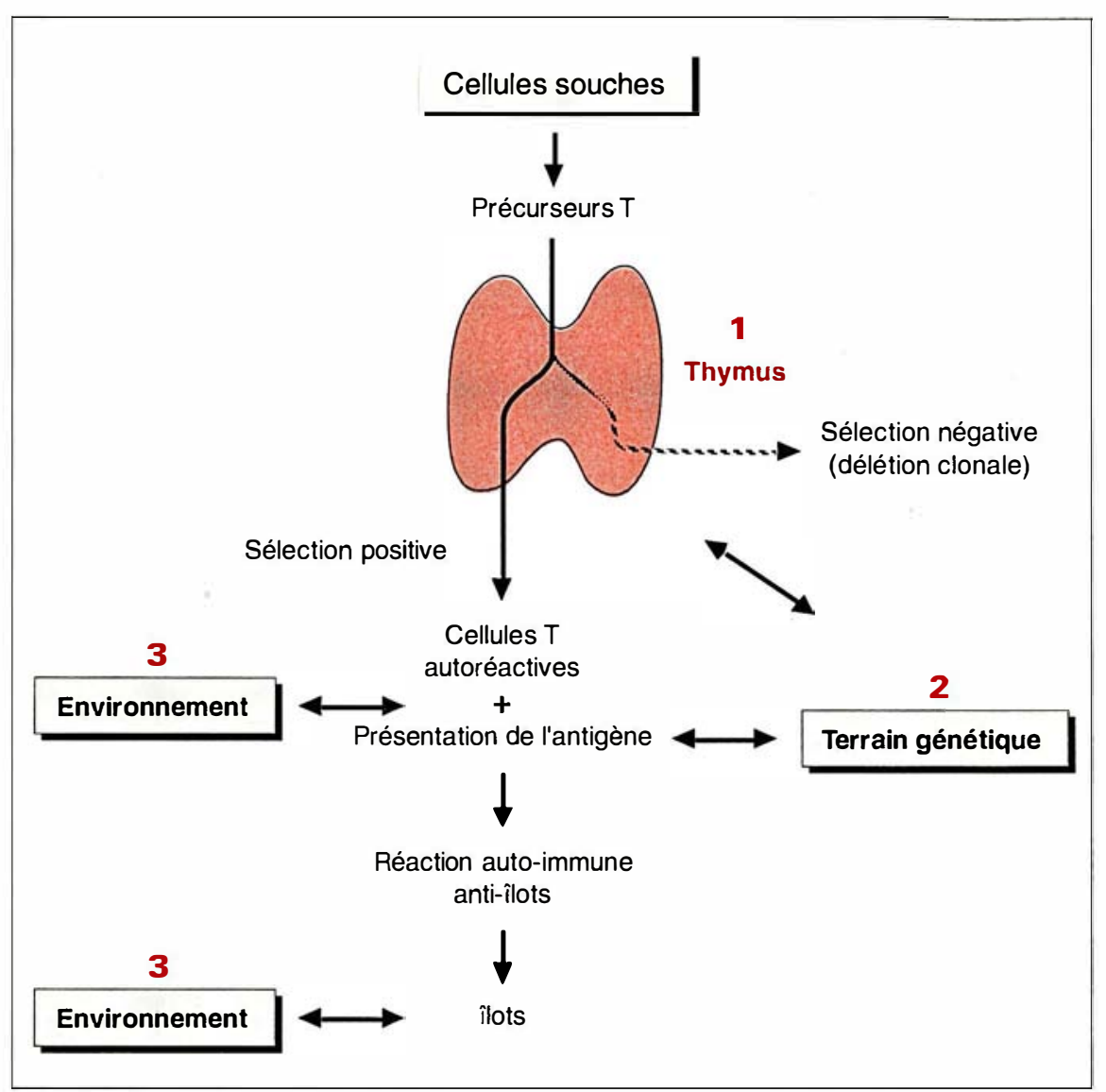

Figure 3. La réaction autoimmune anti-îlots : le rôle du système immunitaire et de l'environnement. 1. La présence de cellules $T$ autoréactives est établie au cours du diabète. La signification pathologique (anomalie de la sélection du répertoire $T$ ) ou physiologique (présence chez tout individu) de la présence des lymphocytes autoréactifs n'est pas connue. 2. Le rôle du terrain génétique est incomplètement connu. On sait que des allèles de classe Il par le CMH sont susceptibles de conditionner la capacité de présentation de l'autoantigène. Le CMH est aussi susceptible d'influencer la sélection du répertoire $T$. 3. L'environnement (virus, toxiques) en interférant avec le système immunitaire ou la cible est susceptible de moduler la survenue de la maladie chez l'animal. Le rôle de l'environnement dans le déclenchement de la réaction autoimmune demeure cependant inconnu.

NOD et du rat BB est prévenu par toutes les interventions visant les cellules $\mathrm{T}$ et le diabète de la souris NOD est transférable par des cellules $T$ purifiées à des receveurs nouveau-nés rendus agammaglobulinémiques à la suite d'un traitement périnatal par un sérum anti-IgM.

Les anticorps anti-îlots, marqueurs précoces de la maladie. La détection d'anticorps circulants dirigés contre les cellules insulaires par G.F. Bottazzo en 1974 a été un argument de poids en faveur de l'origine autoimmune du diabète [24]. Ces anticorps sont détectés par immunofluo- rescence indirecte sur coupes de pancréas humain sur lesquelles ils reconnaissent des antigènes cytoplasmiques. L'expression des antigènes reconnus par les cellules $\beta$, mais aussi par les cellules à glucagon et à somatostatine, va contre un rôle direct de ces anticorps dans la destruction sélective des cellules insulinosécrétrices qui est la marque métabolique et histologique de la maladie. Ces anticorps constituent néanmoins un marqueur biologique de référence utilisable dans un but diagnostique. Des anticorps plus sélectivement dirigés contre les cellules $\beta$, reconnais- 


\section{RÉFÉRENCES}

20. Cohen D, Cohen O, Marcadet A, et al. Class II-HLA-DC $\beta$-chain DNA restriction fragments differentiate among HLA-DR2 individuals in insulin-dependent diabetes and multiple sclerosis. Proc Natl Acad Sci USA 1984; 81 : 1774-8.

21. Todd JA, Acha-Orbea H, Bell JI, et al. A molecular basis of MHC class IIassociated immunity. Science $1988 ; 240$ : 1003-9.

22. Khalil I, d'Auriol L, Gobet M, et al. A combination of HLA-DQ $\beta$ Asp 57-negative and HLA DQ $\alpha$ Arg52 confers susceptibility to insulin-dependent diabetes mellitus. J Clin Invest $1990 ; 85$ : 1315-9.

23. Prochazka M, Leiter EH, Serreze DV Coleman DL. Three recessive loci required for insulin-dependent diabetes in nonobese diabetic mice. Science $1987 ; 237: 286-9$.

24. Bottazzo GF, Dean BM, McNally JM, MacKey EH, Swift PGF, Camble DR. In situ characterization of autoimmune phenomena and expression of HLA molecules in the pancreas in diabetic insulitis. $N$ Engl J Med 1985 ; 313 : 353-60.

25. Palmer JP, Asplin CM, Clemons P. et al. Insulin antibodies in insulin-dependent diabetic before insulin treatment. Science 1983 ; 222 : 1337-9.

26. Gepts W, Lecompte PM. The pancreatic islets in diabetes. Am J Med 1981; 70 : 105-15.

27. Bottazzo GF, Florin-Christenson A, Doniach D. Islet-cell antibodies in diabetes mellitus with autoimmune polendocrine deficiencies. Lancet 1974 : ii : 1279-82.

28. Elias D, Markovits D, Reshef T, Van der Zee R, Cohen IR. Induction and therapy of autoimmune diabetes in the nonobese diabetic (NOD/Lt) mouse by a $65 \mathrm{kDa}$ a heat shock protein. Proc Nall Acad Sci USA 1990 ; 87 : 1576-80.

29. Mandrup-Poulsen $T$, Bendtzen $K$, Dinarello CA, Nerup J. Human tumor necrosis factor potentiates human interleu kin I-mediated rat pancreatic beta cell toxi- sant des antigènes de surface, ont été détectés sur des suspensions de cellules d'îlots.

On peut rapprocher des anticorps anti-cellules d'îlots les auto-anticorps anti-insuline observés avant toute insulinothérapie avec une prévalence voisine de $30 \%$, surtout chez les sujets jeunes [25]. Leur signification physiopathologique est mal connue. L'exposition de motifs antigéniques inhabituels de l'insuline peut expliquer leur production. La mise en évidence parallèle d'anticorps antipro-insuline ou la présence d'autoanticorps reconnaissant des antigènes ubiquitaires, en particulier des protéines du cytosquelette (tubuline, actine, réticuline), des antigènes nucléaires (ADN simple brin, ARN) vont dans le même sens.

L'insulite. L'histologie du pancréas au cours du DID associe une réduction de plus de 70-90\% du nombre des cellules $\beta$, au sein d'îlots de Langerhans dont l'architecture est bouleversée, et une infiltration où prédominent les lymphocytes $\mathrm{T}$ (insulite). L'insulite indique qu'une réaction immunitaire se développe au sein des îlots sans qu'on puisse préjuger néanmoins de sa nature auto-immune. Elle a une distribution focale et s'observe au niveau d'îlots comportant encore des cellules $\beta$. Elle est contemporaine de l'éclosion clinique de la maladie [26]. La composition de l'infiltrat dépend du stade de la maladie, ce qui explique probablement en partie les différences observées selon le modèle étudié. Chez l'homme, ce sont des lymphocytes $\mathrm{T} \mathrm{CD} 8+$ qui sont les plus nombreux, coexistant avec un pourcentage plus faible de cellules $T$ $\mathrm{CD}_{4}+$. Une augmentation de l'expression des antigènes de classe II du CMH sur l'endothélium vasculaire et leur expression inattendue sur les cellules $\beta$ elles-mêmes ont été observées [27]. Chez le rat $\mathrm{BB}$, des biopsies itératives ont montré l'installation de l'insulite jusqu'à 18 jours avant l'éclosion du diabète clinique et la présence initiale de macrophages. Chez la souris NOD, l'infiltrat est composé en majorité des cellules $\mathrm{CD}^{+}+$, en minorité de cellules CD8 +, recrutées à un stade tardif. L'expression des antigènes de classe II par les cellules $\beta$ est mal établie dans les modèles animaux.
Cellules $\mathrm{T}$ activées. Les diabétiques présentent, au début de leur maladie, un nombre anormalement élevé de cellules $T$ activées, reconnues par la présence de marqueurs membranaires d'activation (antigènes DR, récepteur de l'interleukine 2). Ccs cellules activées sont à la fois retrouvées dans le sang circulant et dans l'insulite. Il est difficile de dire, néanmoins, si leur présence correspond à des cellules $T$ spécifiquement engagées dans la réponse auto-immune anti-îlots ou traduisent une activation plus générale, non spécifique, du système immunitaire.

Cellules $\mathbf{T}$ anti-îlots. La recherche de tests cellulaires détectant des lymphocytes $\mathrm{T}$ spécifiques des cellules $\beta$ est essentielle dans une maladie dont le développement repose sur les lymphocytes T. Plusieurs tests ont été décrits dont aucun n'autorise aujourd'hui une utilisation en pratique clinique. L'utilisation de cellules d'insulinome humain a initialement suggéré l'existence de lymphocytes autoréactifs formant des rosettes spécifiques. Plus récemment, des lymphocytes $\mathrm{T}\left(\mathrm{CD} 4+\right.$ et $\left.\mathrm{CD} 8{ }^{+}\right)$ formant des rosettes avec des cellules d'insulinomes de rat ont été observés en nombre accru chez les sujets diabétiques. Une cytotoxicité des lymphocytes de sujets diabétiques a de même été observée vis-à-vis de cellules d'insulinomes. La démonstration d'une action inhibitrice des lymphocytes circulants de sujets diabétiques sur la fonction endocrine de cellules d'îlots de Langerhans murines isolées in vitro a également été rapportée sur la base d'un test fonctionnel d'inhibition de la secrétion d'insuline. Cette action inhibitrice est spécifique des cellules $\beta$ et elle est relayée par des lymphocytes $T$. Ces tests cellulaires, dans les conditions où ils sont utilisés, soulèvent le problème théorique non résolu de l'absence de restriction évidente par les antigènes du $\mathrm{CMH}$. Le rôle de lymphokines a pu être avancé.

On peut espérer que des tests plus informatifs deviendront accessibles lorsque des antigènes d'îlots auront été identifiés. Des résultats récemment obtenus par I. Cohen suggèrent que la protéine de choc thermique de poids moléculaire $65 \mathrm{kDa}$ pourrait présenter une réaction croisée avec 
un des antigènes cibles des cellules d'îlots [28]. En tout état de cause, la production de clones de cellules $\mathrm{T}$ spécifiques d'antigènes d'îlots chez la souris NOD indique la réalité de ces cellules $\mathrm{T}$ anti-îlots et laisse espérer la mise au point de tests cliniques.

Cellules $\mathbf{T}$ effectrices. Plusieurs types de mécanismes effecteurs sont susceptibles d'être impliqués dans la destruction des cellules $\beta$ des îlots de Langerhans. Si une cytotoxicité relayée par des auto-anticorps (cytotoxicité complément-dépendante, cytotoxicité cellulaire dépendante d'anticorps ou ADCC) a été objectivée sur des cellules insulaires in vitro, des arguments convergents indiquent qu'il ne s'agit pas d'un mécanisme important in vivo. Des données récentes indiquent la sensibilité privilégiée des cellules $\beta$ à l'action cytotoxique de l'interleukine 1 [29]. La place réelle de cytokines dans la destruction des cellules $\beta$ au cours du diabète reste néanmoins controversée. Elle est mal établie in vivo, en particulier dans les modèles animaux $[30,31)$. Le rôle des lymphocytes $T$ paraît devoir être privilégié, même s'il n'a pu être définitivement démontré chez l'homme. La présence de lymphocytes $\mathrm{T}$ cytotoxiques vis-à-vis des cellules $\beta$ marquées, restreints par les antigènes de classe $I$, de phénotype CD8 +, a été démontrée chez la souris [32].

Cellules $\mathbf{T}$ régulatrices. Comme toute réponse immunitaire, la réaction auto-immune anti-îlots est soumise à une régulation fine et complexe par les cellules T " auxiliaires " et " suppressives ". La question se pose, dans le diabète comme dans toutes les autres maladies auto-immunes, du rôle physiopathologique d'un éventuel déficit des mécanismes régulateurs impliqués. De façon plus générale, la perturbation de mécanismes régulateurs capables de maintenir à l'état inactivé les clones autoréactifs (présents à l'état physiologique ou pathologique) doit être avancée pour expliquer le déclenchement de la réaction autoimmune anti-îlots. Plusieurs mécanismes peuvent être en jeu : (1) un déficit T suppresseur ; (2) la perturbation d'un réseau d'idiotypes et d'anti-idiotypes en équilibre $(\mathrm{m} / \mathrm{s}$ supplément au $n^{\circ} 1$, vol. 5, p. 13); (3) une modification de l'expression d'auto-antigènes ou de leur présentation au système immunitaire. Aucun argument expérimental n'a permis, à ce jour, de trancher entre ces mécanismes, qui ne sont pas mutuellement exclusifs. Si la détection d'anticorps anti-insuline et anti-récepteurs de l'insuline, chez l'homme, est susceptible de s'intégrer à un schéma de type idiotype-anti-idiotype, rien ne place à ce jour l'insuline ou son récepteur au premier plan des antigènes cibles dans cette maladie. Des arguments importants en faveur d'un déficit en cellules suppressives ont été apportés chez le rat BB [33] et chez la souris NOD [34]. Un diabète est déclenché chez le rat $\mathrm{BB}$ par l'injection d'anticorps reconnaissant une sous-population de lymphocytes $\mathrm{T}$ $\left(\mathrm{RT}^{+}{ }^{+}\right)$dont les expériences de transfert démontrent qu'ils fonctionnent comme des cellules suppressives. On peut rapprocher de ces observations la prévention du diabète du rat $\mathrm{BB}$ par l'injection de cellules sanguines ou médullaires d'animaux normaux, compatible avec le rôle des cellules suppressives. Des observations voisines ont été rapportées chez la souris NOD, chez laquelle ont pu être définies des cellules $\mathrm{T}$ suppressives $\mathrm{CD} 4{ }^{+}$. L'injection à des souris NOD irradiées de cellules de souris NOD non diabétiques bloque le transfert du diabète par injection de cellules spléniques de souris diabétiques.

Les données disponibles chez l'homme sur le rôle des lymphocytes $\mathrm{T}$ "régulateurs" sont indirectes. Elles reposent sur la numération des populations lymphocytaires dans le sang circulant ou sur l'étude des fonctions suppressives. Une augmentation du rapport des lymphocytes $\mathrm{T}$ CD4 + sur les lymphocytes T CD8 + peut être observée au moment de l'éclosion clinique de la maladie. Il existe par ailleurs une diminution de l'activité non spécifique des lymphocytes $T$ suppresseurs induite par la concanavaline $\mathrm{A}$ in vitro. La signification de ces modifications cellulaires demeure incertaine en l'absence de spécificité vis-à-vis des îlots de Langerhans.

Répertoire des cellules $\mathrm{T}$ anti-îlots. Les cellules $\mathrm{T}$ effectrices et régulatrices spécifiques des antigènes d'îlots ont en commun d'utiliser un récepteur pour l'antigène constitué d'un hétérodimère $a \beta$. La question se pose de savoir si les clones pathogènes ou régulateurs ont une utilisation restreinte de certains gènes $\mathrm{V} \alpha$ ou $\mathrm{V} \beta$ du récepteur comme cela paraît être le cas dans l'encéphalomyélite allergique expérimentale. Des données préliminaires obtenues par C. Carnaud indiquent que le gène $V \beta 6$ pourrait être utilisé de façon préférentielle, ce qui ouvrirait d'importantes perspectives - fondamentales et thérapeutiques.

\section{Conclusions}

L'activation du système immunitaire suppose la préexistence de clones lymphocytaires autoréactifs. Deux voies d'activation sont possibles. La première passe par l'activation initiale de lymphocytes $\mathrm{T}$ par les déterminants d'un antigène étranger (par exemple, un agent infectieux) et la stimulation secondaire de lymphocytes effecteurs spécifiques de déterminants donnant une réaction croisée avec un auto-antigène. Aucun argument indiquant l'existence d'une réactivité croisée entre des antigènes insulaires et des antigènes étrangers n'a, à ce jour, été apporté en faveur d'une telle hypothèse. Le rôle central des lymphocytes $\mathrm{T}$ au cours du diabète, l'isolement de clones $\mathrm{T}$ autoréactifs chez la souris NOD permettent de postuler une deuxième voie : l'activation directe de clones $\mathrm{T}$ autoréactifs spécifiques d'antigènes insulaires. La question demeure au cours du diabète, et en auto-immunité en général, de la signification des lymphocytes $T$ autoréactifs qui sont mis en jeu. On peut imaginer qu'ils sont présents chez tout individu, mais que leur activation nécessite un terrain génétique favorable ou l'intervention de facteurs d'environnement. On peut aussi imaginer que leur présence résulte d'une anomalie dans la sélection du répertoire de lymphocytes $\mathrm{T}$ au niveau du thymus.

Le rôle possible d'une modification de la présentation d'auto-antigènes membranaires ressort d'observations qu'aucun argument expérimental définitif n'a permis de confirmer. L'expression pathologique d'antigènes de classe II du CMH par les cellules $\beta$ a été rapportée comme suscep- 


\section{RÉFÉRENCES}

30. Jacob CO, Aiso S, Michie SA, McDevitt $\mathrm{HO}$, Acha-Orbea $\mathrm{H}$. Prevention od diabetes in nonobese diabetic mice by tumor necrosis factor (TNF) : similarities between TNF- $\alpha$ and interleukin 1. Proc Nall Acad Sci USA 1990 ; 87 : 968-72.

31. Sutton R, Gray DWR, McShane P, Dallman MJ, Morris PJ. The specificity of rejection and the absence of susceptibility of pancreatic islet $b$ cells to nonspecific immune destruction in mixed strain islets grafted beneath the renal capsule in the rat. J Exp Med $1989 ; 170$ : 751-62.

32. Nagata M, Yokono K, Hayakawa M, et al. Destruction of pancreatic islet cells by cytotoxic $\mathrm{T}$ lymphocytes in nonobese diabetic mice. $J$ Immunol $1989 ; 143: 1155-62$.

33. Greiner DL, Mordes JP, Handler ES, Angelillo M, Nakamura N, Rossini AA. Depletion of RT6 + lymphocytes induces diabetes in resistant biobreeding/Worcester (BB/W) rats. $J$ Exp Med 1987 ; 166 : 461-5.

34. Boitard C, Yasunami R, Dardenne M, Bach JF. T-cell mediated inhibition of the transfer of autoimmune diabetes in NOD mice. J Exp Med 1989 : 169 : 1669-80.

35. Markmann J, Lo D, Naji A, Palmiter RD, Brinster RL, Heber-Katz E. Antigen presenting function of class II MHC expressing pancreatic beta cells. Nalure 1988 ; 336 : 476-9.

36. Beakkeskov S, Aanstoot HJ, Christgau $\mathrm{S}$, el al. Identification of the $64 \mathrm{~K}$ autoantigen in insulin-dependent diabetes as the GABA-synthetizing enzyme glutamic acid decarboxylase. Nalure $1990 ; 347: 151-6$.

37. Nayak RC, Omar MAK, Rabizadeh A, Srikanta S, Eisenbarth GS. "Cytoplasmic " islet cell antibodies. Evidence that the target antigen is a sialoglycoconjugate. Diabetes $1985 ; 34: 617-9$.

38. Roep B, Arden SD, de Vries RRP, Hutton JC. T-cell clones from a type-1 diabetes patient respond to insulin secretory tible d'induire la présentation d'autoantigènes coexprimés par ces cellules. Les modèles de souris transgéniques où l'expression par les cellules $\beta$ $d$ 'antigènes de classe II ne conduit pas à une réaction immunitaire antiîlots, voire induit une tolérance immunitaire [35], vont contre un rôle dans le déclenchement du diabète. On peut néanmoins remarquer que les antigènes de classe II ainsi greffés sur les cellules $\beta$ ne sont pas ceux dont on a rapporté chez la souris une association au diabète (I-A NOD en particulier).

Il est important de souligner que la nature biochimique de l'antigène cible de la réaction auto-immune anti-îlots reste inconnue. L'utilisation de sérums provenant de sujets ayant un DID récent pour précipiter des antigènes insulaires marqués par la méthionine S35 a permis l'identification d'un antigène de poids moléculaire $64 \mathrm{kDa}$ identifié comme la décarboxylase de l'acide glutamique $(\mathrm{m} / \mathrm{s}$ no 9, vol. 6, p. 921) [36]. D'autres approches ont été utilisées. La technologie des hybridomes a permis l'obtention d'anticorps monoclonaux spécifiques des cellules insulaires et la caractérisation des antigènes cibles spécifiques sur les cellules insulaires. Ont ainsi été identifiés des auto-antigènes insulaires dont aucun n'a conduit, à ce jour, à la preuve expérimentale de son rôle dans le développement d'un diabète. Des anticorps circulants dirigés contre un glycolipide ont été caractérisés au cours du DID [37]. Des anticorps monoclonaux ont été obtenus chez le rat $\mathrm{BB}$ et la souris NOD qui reconnaissent d'autres antigènes cibles possibles sur les cellules insulaires. Enfin, des clones de lymphocytes $\mathrm{T}$ spécifiques d'une protéine de $38 \mathrm{kDa}$ - des granules de sécrétion des cellules $\beta-$ ont été individualisés chez l'homme [38].

La participation directe d'une réaction auto-immune à la destruction des cellules $\beta$ des îlots de Langerhans du pancréas est établie. Elle apporte dès à présent des paramètres biologiques susceptibles de permettre un diagnostic précoce de la maladie. Elle suggère aussi une nouvelle approche du traitement de la maladie, visant directement le processus immunitaire responsable de son développement

\section{Summary}

Physiopathology of insulindependent diabetes mellitus, a polygenic autoimmune disease

Insulin-dependent diabetes mellitus is an autoimmune disease. It develops on a suceptibility background which has been partly characterized. One suceptibility gene is localized within the major histocompatibility complex in the $D$ region which encodes class II antigens. It is likely to correspond to DQ alleles in the human. The localization of other suceptibility genes remains unknown. The development of clinical diabetes follows a progressive infiltration of the islets of Langerhans of the pancreas by lymphoid cells, mostly $\mathrm{T}$ lymphocytes. Despite the detection of islet cell antibodies in the serum of patients with recent onset diabetes, $\mathrm{T}$ cells play a predominant role in the discase process. Relevant models for autoimmune diabetes have been developed in the mouse (the NOD mouse) and the rat (the $\mathrm{BB}$ rat). The transfer of diabetes has been achieved in both models by injecting purified $T$ cells into non diabetic syngeneic recipients. The development of diabctes is also prevented in both models by monoclonal antibodies directed against $\mathrm{T}$ lymphocytes or class II major histocompatibility complex antigens or by selectively hitting macrophages. The interaction between autoreactive $\mathrm{CD}_{4}+\mathrm{T}$ cells and antigen presenting cells thus appears central to the autoimmune process. However, the primary event responsible for the activation of autoreactive $\mathrm{T}$ cells is still to be understood. Anti-islet autoimmunity can be viewed as a disease of lymphoid cells (e.g. T cells). Alternatively it may result from environmental events triggering activation of the autoimmune process.

\section{TIRÉS A PART}

C. Boitard. 